InternationalTinnitusJournal.2018;22(2):150-155.

\title{
Ageusia, Hyposmia, Dizziness and Tinnitus as Presenting Symptoms of Multiple Sclerosis
}

\author{
Massimo Ralli \\ Vittorio D'Aguanno' \\ Marta Altieri² \\ Arianna Di Stadio ${ }^{3 *}$ \\ Rosaria Turchetta ${ }^{1}$ \\ Marco de Vincentiis ${ }^{4}$ \\ Vittorio Di Piero \\ Antonio $\mathrm{Greco}^{1}$
}

\begin{abstract}
Multiple Sclerosis (MS) is a chronic inflammatory and neurodegenerative disease of the central nervous system characterized by the destruction of myelin sheaths and axonal loss. Presenting symptoms of multiple sclerosis may vary and if not promptly identified may lead to a delayed diagnosis of Multiple sclerosis.

In this paper, we present a rare case of a patient with ageusia as presenting symptom of Multiple sclerosis; the symptom spontaneously resolved after two weeks and was followed by hyposmia, dizziness, tinnitus and facial paraesthesia that eventually led to a clinical diagnosis of Multiple sclerosis.

The presence of gustatory, olfactory and audio-vestibular symptoms has been associated with disease activity and progression of several inflammatory and neurodegenerative diseases of the central nervous system including Multiple sclerosis. Gustatory alterations may be due to the presence of demyelinating lesion in the brain area of taste; the progression of the disease may explain the additional olfactory and audio-vestibular symptoms described in this patient.

Even if taste alteration is an extremely rare presenting symptom of Multiple sclerosis, its presence in young women should always be carefully investigated as it could be an early sign of Multiple sclerosis.
\end{abstract}

Keywords: olfaction, taste, demyelination, multiple sclerosis.

${ }^{1}$ Department of Sense Organs, Sapienza University of Rome, Rome, Italy

${ }^{2}$ Department of Neurology and Psychiatry, Sapienza University of Rome, Rome, Italy

${ }^{3}$ Department of Otolaryngology, University of Perugia, Italy

${ }^{4}$ Department of Odontostomatology and Maxillo facial Sciences, Sapienza University of Rome, Rome, Italy

Send correspondence to:

Arianna Di Stadio

Department of Otolaryngology, University of Perugia, Perugia, Italy. E-mail: ariannadistadio@hotmail.com

Paper submitted to the ITJ-EM (Editorial Manager System) on October 31, 2018; and accepted on November 06, 2018. 


\section{INTRODUCTION}

Multiple Sclerosis (MS) is a chronic inflammatory and neurodegenerative disease of the Central Nervous System (CNS) characterized by the progressive destruction of myelin sheaths and axonal loss, typically presenting in the third and fourth decade of life ${ }^{1}$. The demyelination may affect different areas of brain and medulla and be responsible of different otolaryngologic symptoms including gustatory, olfactory, and audio-vestibular alterations ${ }^{1}$.

MS may alter the olfactory and gustatory functions at both the functional and structural levels ${ }^{2}$; the neurological symptoms may vary depending on the brain region affected by demyelination. The olfactory function is altered in $15 \%$ to $45 \%$ of patients that suffer from $\mathrm{MS}^{3,4}$, while taste is altered in only $4 \%$ of cases. The combination between olfactory and taste alterations is reported in nearly $19 \%$ of MS patient ${ }^{5}$. Based on self-report or cursory sensory testing, MS-related taste disorders have been assumed to be rare6; furthermore, patients reporting taste deficits typically have associated olfactory dysfunction, and may be inaccurate in recognizing true taste dysfunction when present. The smell dysfunction that could increase the taste alteration is progressive and slowly deteriorates over time $e^{4,7,8}$ the increasing of smell dysfunction impacts taste also by increasing the symptom ${ }^{4,5,9,10}$.

Audio-vestibular symptoms, such as sudden, transient or progressive sensorineural hearing loss, tinnitus and dizziness are common in patients with MS, and have a significant impact on quality of life of affected subjects ${ }^{11}$. Audio-vestibular symptoms may arise from central alterations, such as demyelination in the auditory or vestibular pathways, or more rarely can be peripheral, following the involvement of the peripheral audiovestibular system ${ }^{12-14}$.

Olfactory, gustatory and audio-vestibular symptoms are often of temporary nature, probably because of the relapsing/remitting events that occur during the course of MS or following the use of corticosteroids that contrast the systemic inflammation generated by $\mathrm{MS}^{15}$.

In this article, we report the case of a young woman presenting with a complete loss of taste that spontaneously resolved after two weeks, gradually followed by olfactory, audio-vestibular and neurological symptoms that led to a clinical diagnosis of MS.

\section{CASE REPORT}

A 32 years old woman was admitted to the Neurology Department of the Policlinico Umberto I, Sapienza University of Rome, referring the occurrence of a complete loss of taste (ageusia) nearly 3 months before admission with spontaneous resolution two weeks later. Ageusia was followed, about one week after its resolution, by hyposmia, dizziness and tinnitus in the absence of subjective hearing loss, and present at admission. One week before admission, the patient also experienced the loss of strength in the left side of the body and facial paraesthesia that led the patient to seek for medical help. In her medical history, she denied previous neurological disease and referred insulin resistance in treatment with metformin and hypothyroidism. After admission, the patient underwent a comprehensive neurological and otolaryngologic examination.

Neurological examination revealed a slight gaitabnormality and little movements towards the left side when eyes were closed. Tactile sensitivity showed no disturbances at time of the evaluation. Brainstem Auditory Evoked Responses (BAER) Table 1, Motor Evoked Potentials (MEPs) Table 2, Visual Evoked Potentials (VEP) Table 3 was normal.

Otolaryngology examination including nasal endoscopy did not yield to any significant abnormalities. Tonal audiometry demonstrated bilateral normal hearing defined as an individual hearing threshold $<25 \mathrm{~dB} H \mathrm{HL}$ at frequencies from 250 to $4,000 \mathrm{~Hz}$ at the octave scale in both ears according to the American Academy of Otolaryngology and American Council of Otolaryngology Figure 1. The measurement of the characteristics of tinnitus indicated a bilateral continuous non-pulsatile tinnitus with a frequency near $3000 \mathrm{~Hz}$ and an intensity of $20 \mathrm{~dB}$. The Italian versions of the Tinnitus Handicap Inventory $(\mathrm{THI})^{16}$ and Hearing Handicap Inventory $(\mathrm{HHI})^{17}$ questionnaires were administered to the patients. THI score was 24; $\mathrm{HHI}$ score was 4 . Somatic maneuvers were

Table 1: Brainstem auditory evoked responses [Brainstem Auditory Evoked Response recording in the present case. Values were within normal range].

\begin{tabular}{lccccc}
\hline Exam & $\begin{array}{c}\text { Auditory } \\
\text { Stimulus (dB) }\end{array}$ & I (ms) & III (ms) & V (ms) & III-V (ms) \\
$\begin{array}{lccccc}\text { S-BAER } \\
\text { Ipsilateral }\end{array}$ & $75 \mathrm{nHL}$ & 1.74 & 3.92 & 5.7 & 1.78 \\
$\begin{array}{l}\text { Contralateral } \\
\text { D-BAER }\end{array}$ & & & 3.8 & 5.9 & 2.1 \\
$\begin{array}{l}\text { Ipsilateral } \\
\text { Contralateral }\end{array}$ & $75 \mathrm{nHL}$ & 1.88 & 3.96 & 5.94 & 1.98 \\
\hline
\end{tabular}

Table 2: Motor Evoked Responses [Motor Evoked Response recording in the present case. Values were within normal range].

\begin{tabular}{|c|c|c|c|c|c|}
\hline Nerve & $\begin{array}{l}\text { Latency } \\
\text { (ms) }\end{array}$ & $\begin{array}{l}\text { CCT } \\
\text { (ms) }\end{array}$ & & $\begin{array}{l}\text { Latency } \\
\text { (ms) }\end{array}$ & CCT (ms) \\
\hline S-ULNAR ADM & & & \multicolumn{3}{|c|}{ S-TIBIAL MALLEOLUS } \\
\hline Cortical & 20.15 & 10.15 & Cortical & 26.95 & 19.75 \\
\hline Radicolar & 10 & & Radicolar & 7.2 & \\
\hline D-ULNAR ADM & & & \multicolumn{3}{|c|}{ D-TIBIAL MALLEOLUS } \\
\hline Cortical & 19.1 & 9.05 & Cortical & 27.65 & 20.05 \\
\hline Radicolar & 10.1 & & Radicolar & 7.6 & \\
\hline
\end{tabular}

Table 3: Visual Evoked Responses [Visual Evoked Response recording in the present case. Values were within normal range].

\begin{tabular}{ccc}
\hline Stimulus (B/N) & Right eye & Left eye \\
\hline Latency $(\mathrm{ms})$ & Latency $(\mathrm{ms})$ \\
\cline { 2 - 3 } & 109 & 110 \\
\hline Amplitude $(\mathrm{mV})$ & Amplitude $(\mathrm{mV})$ \\
\hline 7.6 & 6.05 \\
\hline
\end{tabular}




\section{Pure Tone Audiometry}

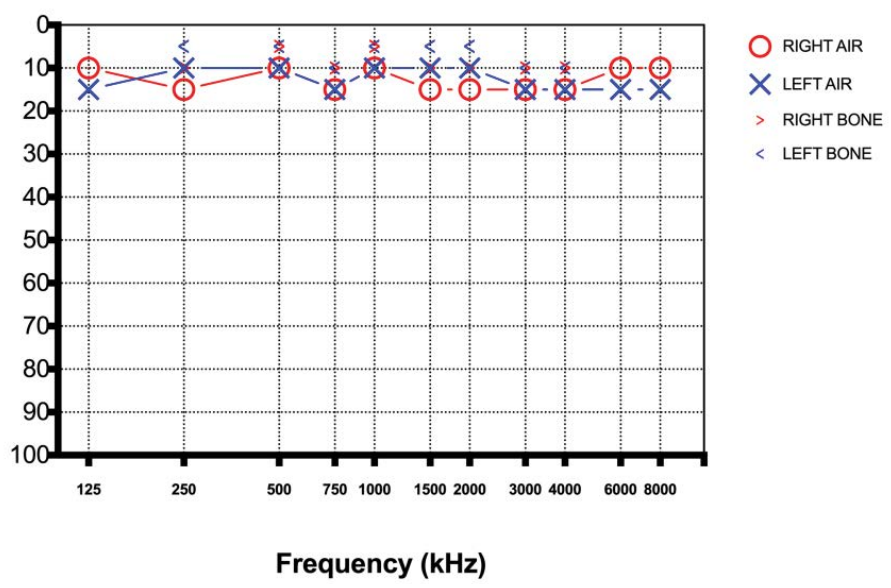

Figure 1: Pure Tone Audiometry showing a bilateral normal hearing.

performed to evaluate the presence somatic tinnitus ${ }^{18-22}$; all maneuvers on the temporomandibular joint and head and neck did not induce a modulation of tinnitus pitch or loudness, thus excluding a somatosensory origin of tinnitus.

Distortion-product Otoacoustic Emissions (DPOAEs) were recorded and were consistent with audiometric findings Figure 2 although a bilateral decrease of DP intensity was recorded for frequencies between 4,358 and $5,188 \mathrm{~Hz}$.

Vestibular evaluation revealed a spontaneous brief right-beating horizontal nystagmus, although FitzgeraldHallpike caloric testing was normal.

In the suspicion of a CNS disease, an MRI of the brain was performed, reveling the presence small areas of hyperintensity in FLAIR sequences in the periventricular space, in the semioval center and at the level of the corona radiata, compatible with multifocal leukoencephalopathy;

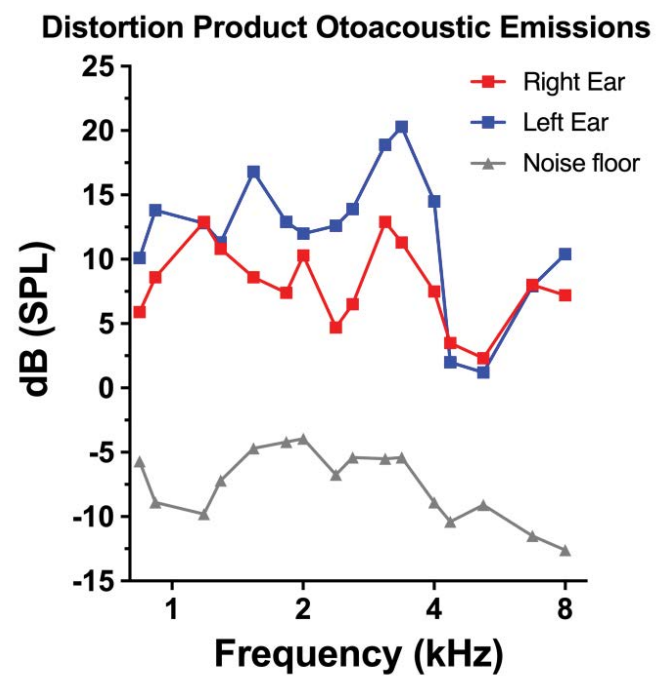

Figure 2: Distortion Product Otoacoustic Emissions were within normal range. similar alterations were observed in the left medium and lower cerebellar peduncles.

Due to clinical and radiologic features suggesting a demyelinating disease, the patients underwent a lumbar puncture. Cerebrospinal Fluid (CSF) analysis showed the presence of oligoclonal bands suggestive of MS.

Further autoimmunity evaluation was performed including testing for antinuclear antibody, double-strand DNA antibody, phospholipid antibody, cardiolipin antibody, antineutrophil cytoplasmic antibody and perinuclear antineutrophil cytoplasmic antibody. All tests were negative.

A diagnosis of relapsing-remitting MS was made, and the patient started an immunomodulatory treatment with solumedrol. Associated symptoms promptly disappeared after the initiation of the treatment. No further relapses were observed for the subsequent 6 months.

\section{DISCUSSION}

Taste, olfactory and audio-vestibular alterations have been recognized as important presenting symptoms in various neurodegenerative and neuroinflammatory CNS conditions ${ }^{23}$ and have been reported in patients with MS.

\section{Taste disorders in MS}

The presence of gustatory alterations, as observed in our patient, represents an often underestimated condition in MS patients because of their rarity as presenting symptoms and because they are often associated to additional smell alterations that may confuse the identification of real true taste dysfunctions by the patients when present ${ }^{24}$.

The impairment of taste perception had been reported in approximately $20 \%$ of MS patients ${ }^{25}$ with elevated electrogustometric thresholds. Patients suffering from MS showed difficulties to identify tastants for all four classic taste qualities on both anterior (VII) and posterior regions of the tongue (IX); this loss of sensibility may be due to the involvement of superior taste pathways that influence taste quality more than perceived strength ${ }^{25}$ Figure 3.

Combarros et al. described the case of a patient lamenting transitory gustatory dysfunction that presented hyperintense, enhanced small lesion in the lateral part of the left midpontine tegmentum at MRl; this finding supports the hypothesis that unilateral pontine lesions may result in ipsilateral gustatory deficits and suggests that gustatory fibers ascend from the solitary nucleus in the medulla up to the homolateral pontine tegmentum without decussating ${ }^{26}$. In a different case, bilateral gustatory alteration has been reported in a patient with a unilateral small lesion, suggesting that the ascending gustatory fibers branch at the level of the lesion in the lower midbrain or in the more caudal part ${ }^{9}$.

In our case, the patient presented a complete loss of taste lasting for two weeks with a spontaneous recovery. 


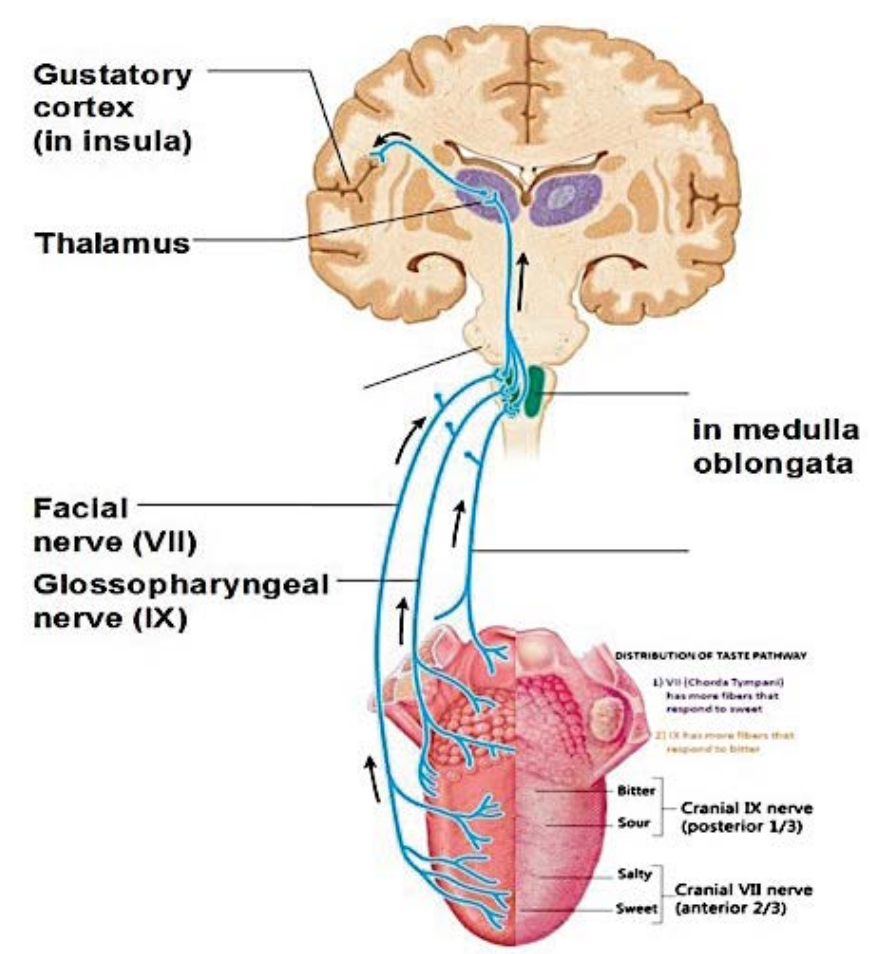

Figure 3: Graphic showing the taste pathways from tongue to the central brain areas.

Ageusia was the only presenting symptom and was not associated with other symptoms, that instead appeared only several weeks later and led the patient to seek for neurological evaluation. In her MRI, areas of demyelination were identified in the semioval center and at the level of the corona radiata that may explain the alteration of taste; in fact, corona radiata in particular has been identified as responsible for taste alteration. The explanation of this phenomenon is linked to the pathways of the gustatory fibers that ascend contralaterally in the cerebral hemisphere and that then ascend from the thalamus to the cerebral cortex via the posterior part of corona radiata.

\section{Smell alterations in MS}

MS patients may present olfactory alterations that lead to odors misidentification and that are mainly present during the active phases of the disease. The MS olfactory dysfunctions are often associated to demyelination in the frontal and temporal lobes ${ }^{7}$. These findings suggest that smell impairment might be an effective indicator of disease progression due to the increasing in number of brain lesions, even in the presence of VEP within normal range ${ }^{23}$.

The incidence of smell disorders in patients with MS is still controversial; percentages range from $15 \%$ to $45 \%$ in case control studies ${ }^{27-31}$. A worse olfactory threshold was observed in the early inflammatory phases while an identification score was more impaired in the widespread chronic disease. The presence of demyelinating plaques observed by MRI in the inferior frontal- and temporal- lobe regions of patients affected from MS is correlated with altered smell abilities and olfactory dysfunctions. As in case of gustatory alterations, the olfactory dysfunction may be an indicator of disease progression due to the increasing of number of lesion or to the extension of demyelinating areas ${ }^{32,33}$.

The mechanisms that link CNS inflammation and olfactory dysfunction are still poorly understood. The smell threshold alteration is considered as associated with the peripheral parts of the olfactory system, while odor discrimination is related to a central process by engaging several brain regions ${ }^{23,24}$. Therefore, in advanced stages of MS, the odor identification may be gradually impaired as the disease progresses in the CNS and may impair the neuronal network required for the processing of odor information 7 . Furthermore, the inflammation in the brain may also affect the peripheral portion of the olfactory system via humoral mechanisms such as cytokine release or antibody-mediated inflammation. In our case, hyposmia was not a presenting symptom and was not associated to ageusia; in fact, its onset followed the spontaneous remission of taste alteration.

\section{Audio-vestibular alterations in MS}

Audio-vestibular symptoms such as hearing loss, tinnitus and vertigo have been often reported in patients with MS. Audio-vestibular alterations, especially in young subjects, could represent an early sign of MS even when no demyelinated plaques are visible in the central nervous system $^{13}$.

Tinnitus in MS patients is most probably a consequence of hearing impairment that affects between $1 \%$ and $17 \%$ of MS patients and is commonly attributed to central nervous system demyelination ${ }^{34}$. Hearing loss and tinnitus may be the only presenting symptom of MS and may appear early in the course of the disease with good prognosis and little or no residual hearing deficit ${ }^{13,15}$.

In the present case, tinnitus was not associated to clinically evident hearing loss; it has been previously reported that lesions affecting the auditory pathways may not determine an evident hearing loss in some cases ${ }^{15}$. Moreover, tinnitus and difficulties in speech perception, especially in noise, are often reported even in the absence of a clinically evident hearing loss ${ }^{35,36}$. Traditional theories assume that tinnitus is triggered by cochlear damage, mainly at the outer hair cell level; however, this would not apply to tinnitus patients presenting with a normal audiogram $^{36,37}$. Recent findings have shown that normal hearing thresholds may also be found in patients with cochlear damage that predominantly affects specific structures, such as the inner hair cells, the auditory nerve fibers and the synapses between them ${ }^{38,39}$. An alteration of these cell populations, especially in the high-frequency region of the cochlea, may configure the presence of hidden hearing loss that cannot be recorded using the clinical audiometry ${ }^{38-41}$. 
Vertigo affects $7 \%$ to $30 \%$ of patients with MS and is mainly attributed to demyelination of the vestibular nuclei, musculoskeletal weakness, and ocular diseases ${ }^{11}$. The most typical vestibular sign in MS patients is multidirectional nystagmus without latency ${ }^{42}$; dizziness and disequilibrium may be also present, while acute vertigo episodes are rarer. In these cases, differential diagnosis with peripheral vestibular disorders is of paramount importance; clinical signs such as adaptation and fatigue of nystagmus, and instrumental tests such as vestibular evoked myogenic potentials may play a role for the differential diagnosis ${ }^{42}$.

Recent evidences of the presence of macrophages in human temporal bones of patients with autoimmune disease supports the hypothesis that inflammation mechanisms in MS may also affect the inner ear leading to growing pathophysiological evidence of the involvement of the inner ear and vestibulocochlear nerve in hearing loss and vertigo in MS patients that may manifest with sudden sensorineural hearing loss and acute vertigo ${ }^{43}$.

Audio-vestibular symptoms in young, neurologically normal subjects, especially when spontaneous recovery occurs, may represent an early sign of MS; it is therefore recommended to evaluate these subjects with clinical, radiological and electrophysiological test to exclude peripheral incipient $\mathrm{MS}^{13,44}$.

\section{CONCLUSION}

In the present case, the presenting symptom of MS was a two-week long ageusia that spontaneously resolved without medical intervention. After its remission, ageusia was followed by other otolaryngologic symptoms such as olfactory and audio-vestibularalterations. Thesesymptoms have been rarely reported, especially when simultaneous, in the early phase of MS and in young patients. A careful prompt investigation with clinical, radiological, and electrophysiological tests are recommended in patients with similar symptoms, especially in case of spontaneous recovery, as they may be presenting symptoms of a CNS disease such as an incipient MS.

\section{REFERENCES}

1. Polman $\mathrm{CH}$. Diagnostic criteria for multiple sclerosis: 2010 revisions to the McDonald criteria. Ann Neurol. 2011;69(2):292-302.

2. DeLuca GC. Olfactory pathology in central nervous system demyelinating diseases. Brain Pathol. 2015;25(5):543-51.

3. Silva AM, Santos E, Moreira I, Bettencourt A, Coutinho E, Goncalves $A$, et al. Olfactory dysfunction in multiple sclerosis: association with secondary progression. Mult Scler. 2012;18(5):616-21.

4. Uecker FC, Olze H, Kunte H, Gerz C, Göktas O, Harms L, et al. Longitudinal testing of olfactory and gustatory function in patients with multiple sclerosis. PLoS One. 2017;12(1):e0170492.

5. Fleiner F, Dahlslett SB, Schmidt F, Harms L, Goektas O. Olfactory and gustatory function in patients with multiple sclerosis. Am J Rhinol Allergy. 2010;24(5):e93-7.

6. Kahana EU, Leibowitz, Alter M. Brainstem and cranial nerve involvement in multiple sclerosis. Acta Neurol Scand. 1973;49(3):269-79.
7. Lutterotti A, Vedovello M, Reindl M, Ehling R, DiPauli F, Kuenz B, et al. Olfactory threshold is impaired in early, active multiple sclerosis. Mult Scler. 2011;17(8):964-69.

8. Bsteh G, Hegen H, Ladstätter F, Berek K, Amprosi M, Wurth S, et al., Change of olfactory function as a marker of inflammatory activity and disability progression in MS. Mult Scler. 2017; p:1352458517745724.

9. Hashimoto T, Doden T, Ono Y, Uematsu T. Bilateral hypogeusia caused by a small lesion in the lower midbrain tegmentum. BMJ Case Rep. 2012.

10. Rollin H. Gustatory disturbances in multiple sclerosis. Laryngol Rhinol Otol. 1976;55(8):678-81.

11. Marrie RA, Cutter GR, Tyry T. Substantial burden of dizziness in multiple sclerosis. Mult Scler Relat Disord. 2013;2(1):21-8.

12. Pula JH, Toker DE, Kattah JC. Multiple sclerosis as a cause of the acute vestibular syndrome. J Neurol. 2013;260(6):1649-54.

13. Di Stadio A, Ralli M. Inner ear involvement in multiple sclerosis: An underestimated condition? Mult Scler. 2018;p:1352458518750010.

14. Frohman EM, Ramer PD, Dewey RB, Kramer L, Frohman TC Benign paroxysmal positioning vertigo in multiple sclerosis: diagnosis, pathophysiology and therapeutic techniques. Mult Scler. 2003;9(3):250-5.

15. Di Stadio A, Dipietro L, Ralli M, Meneghello F, Minni A, Greco A, et al. Sudden hearing loss as an early detector of multiple sclerosis: a systematic review. Eur Rev Med Pharmacol Sci. 2018;22(14):46114624.

16. Passi S, Ralli G, Capparelli E, Mammone A, Scacciatelli D, Cianfrone G. The THI questionnaire: psychometric data for reliability and validity of the Italian version. Int Tinnitus J. 2008;14(1):26-33.

17. Ventry IM, Weinstein BE. The hearing handicap inventory for the elderly: a new tool. Ear Hear. 1982;3(3):128-34.

18. Ralli M, Altissimi G, Turchetta R, Mazzei F, Salviati M, Cianfrone F, et al. Somatosensory Tinnitus: Correlation between Cranio-CervicoMandibular Disorder History and Somatic Modulation. Audiol Neurootol. 2016;21(6):372-382.

19. Ralli M, Greco A, Boccassini A, Altissimi G, Paolo CD, Falasca $\mathrm{V}$, et al. Subtyping patients with somatic tinnitus: Modulation of tinnitus and history for somatic dysfunction help identify tinnitus patients with temporomandibular joint disorders. PLoS One. 2018;13(8):e0202050.

20. Ralli M, Greco A, Cialente F, Stadio AD, Virgilio AD, Longo L, et al. Somatic Tinnitus. Int Tinnitus J. 2017;21(2):112-121.

21. Ralli M, Greco A, Turchetta R, Altissimi G, Vincentiis MD, Cianfrone $G$, et al. Somatosensory tinnitus: Current evidence and future perspectives. J Int Med Res. 2017;45(3):933-947.

22. Won JY, Yoo S, Lee SK, Choi HK, Yakunina N, Le Q, et al. Prevalence and factors associated with neck and jaw muscle modulation of tinnitus. Audiol Neurootol. 2013;18(4):261-73.

23. Moscavitch SD, Szyper-Kravitz M, Shoenfeld Y. Autoimmune pathology accounts for common manifestations in a wide range of neuro-psychiatric disorders: the olfactory and immune system interrelationship. Clin Immunol. 2009;130(3):235-43.

24. Soter A, Kim J, Jackman A, Tourbier I, Kaul A, Doty RL. Accuracy of self-report in detecting taste dysfunction. Laryngoscope. 2008;118(4):611-7.

25. Doty RL, Tourbier IA, Pham DL, Cuzzocreo JL, Udupa JK, Karacali B, et al. Taste dysfunction in multiple sclerosis. J Neurol. 2016;263(4):677-88.

26. Juan S, Berciano J, De Pablos C. Hemiageusia from an ipsilatera multiple sclerosis plaque at the midpontine tegmentum. J Neurol Neurosurg Psychiatry. 2000;68(6):796.

27. Doty RL. Olfactory dysfunction in multiple sclerosis. Relation to 
plaque load in inferior frontal and temporal lobes. Ann N Y Acad Sci. 1998. 855: p. 781-6.

28. Hawkes $\mathrm{CH}$, Shephard BC. Olfactory evoked responses and identification tests in neurological disease. Ann $\mathrm{N}$ Y Acad Sci. 1998;855:608-15.

29. Zivadinov R. Olfactory loss in multiple sclerosis. J Neurol Sci. 1999;168(2):127-30.

30. Zorzon M. Olfactory dysfunction and extent of white matter abnormalities in multiple sclerosis: a clinical and MR study. Mult Scler. 2000;6(6):386-90.

31. Lucassen EB. Olfactory dysfunction in Multiple Sclerosis: A scoping review of the literature. Mult Scler Relat Disord. 2016;6:1-9.

32. Ciurleo R. Olfactory dysfunction as a prognostic marker for disability progression in Multiple Sclerosis: An olfactory event related potential study. PLoS One. 2018;13(4):e0196006.

33. Pimentel ML. Olfactory dysfunction as a marker of multiple sclerosis progression. Arq Neuropsiquiatr. 2016;74(9):693-694.

34. Hellmann MA, Steiner I, Mosberg-Galili R. Sudden sensorineural hearing loss in multiple sclerosis: clinical course and possible pathogenesis. Acta Neurol Scand. 2011;124(4):245-9.

35. Song K. Audiometric profiles in patients with normal hearing and bilateral or unilateral tinnitus. Otol Neurotol. 2018;39(6):e416-e421.

36. Schaette R, McAlpine D. Tinnitus with a normal audiogram: physiological evidence for hidden hearing loss and computational model. J Neurosci. 2011;31(38):13452-7.

37. Eggermont JJ. The auditory cortex and tinnitus-a review of animal and human studies. Eur J Neurosci. 2015;41(5):665-76.

38. Kujawa SG, Liberman MC. Synaptopathy in the noise-exposed and aging cochlea: Primary neural degeneration in acquired sensorineural hearing loss. Hear Res. 2015;330(Pt B):191-9.

39. Liberman MC, Kujawa SG. Cochlear synaptopathy in acquired sensorineural hearing loss: Manifestations and mechanisms. Hear Res. 2017;349:138-147.

40. Ralli M. Tone-in-noise detection deficits in elderly patients with clinically normal hearing. Am J Otolaryngol. 2018.

41. Viana LM. Cochlear neuropathy in human presbycusis: Confocal analysis of hidden hearing loss in post-mortem tissue. Hear Res. 2015;327:78-88.

42. Stys PK. Multiple sclerosis: autoimmune disease or autoimmune reaction? Can J Neurol Sci. 2010;37 Suppl 2:S16-23.

43. O'Malley JT, Nadol JB, McKenna MJ. Anti CD163+, Iba1+, and CD68+ Cells in the adult human inner ear: Normal distribution of an unappreciated class of macrophages/microglia and implications for inflammatory otopathology in humans. Otol Neurotol. 2016;37(1):99108.

44. Furst M, Levine RA. Hearing disorders in multiple sclerosis. Hand Clin Neurol. 2015;129:649-65. 